\title{
Coadministration with Tea Polyphenols Enhances the Neuroprotective Effect of Defatted Walnut Meal Hydrolysate against Scopolamine- Induced Learning and Memory Deficits in Mice
}

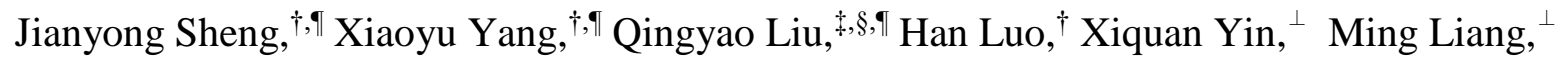
Wei Liu,,${ }^{\dagger}$ Xiaoli Lan, ${ }^{*}, \$$ Jiangling Wan,,${ }^{*} \dagger$ and Xiangliang Yang ${ }^{\dagger}$

†National Engineering Research Center for Nanomedicine, College of Life Science and Technology, Huazhong University of Science and Technology, Wuhan, Hubei 430074, China

¥ Department of Nuclear Medicine, Union Hospital, Tongji Medical College, Huazhong University of Science and Technology, Wuhan, Hubei 430022, China

${ }^{\S}$ Hubei Province Key Laboratory of Molecular Imaging, Wuhan, Hubei 430022, China

${ }^{\perp}$ Joint Laboratory for The research of Modern Preparation Technology-Huazhong University of Science and Technology and Infinitus, Guangzhou, Guangdong 510663, China

* Corresponding author. Wei Liu, Tel: +86-27-87792147. Fax: +86-27-87792234. E-mail: wliu@hust.edu.cn

* Corresponding author. Xiaoli Lan, Mobile: +86-13886193262. E-mail: 1x1730724@ hotmail.com

* Corresponding author. Jiangling Wan, Tel: +86-27-67849580. Fax: +86-27-87792234. E-mail: wanjl@hust.edu.cn

II Jianyong Sheng, Xiaoyu Yang and Qingyao Liu contributed equally to this work. 


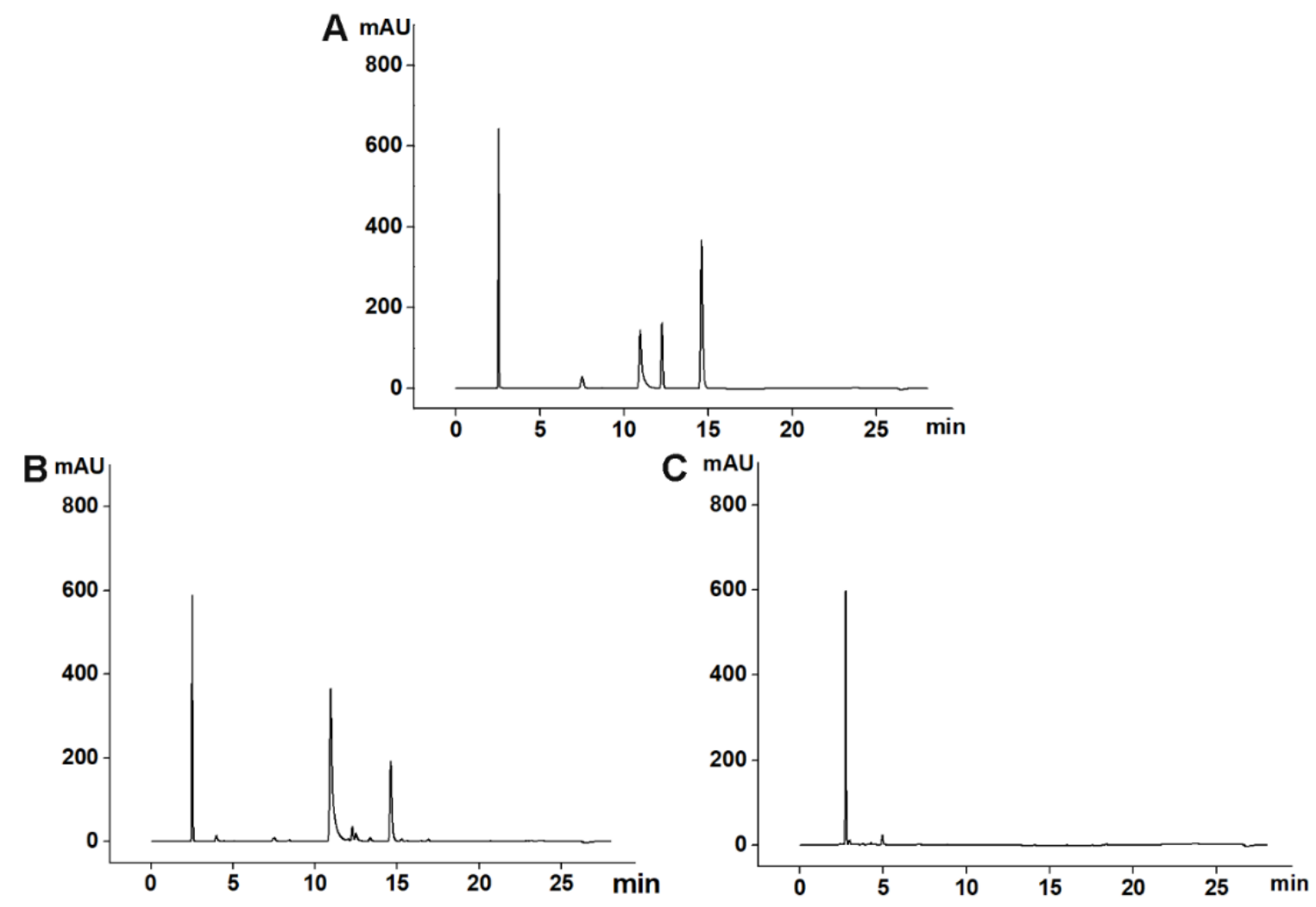

Figure S1. HPLC assay for epicatechin (EC), epicatechin gallate (ECG), epigallocatechin (EGC) and epigallocatechin gallate (EGCG) in TP and DWMH. HPLC chromatograms of the mixture of reference standard of EC, ECG, EGC and EGCG (A), TP (B) and DWMH (C). The retention times for EGC, EGCG, EC and ECG were $7.5 \mathrm{~min}, 11.0 \mathrm{~min}, 12.3 \mathrm{~min}$ and $14.6 \mathrm{~min}$, respectively.

Method: Reference standards or TP were dissolved in $80 \%$ methanol solution and diluted with $10 \%$ methanol solution containing $0.5 \mathrm{mg} / \mathrm{mL}$ ascorbic acid. DWMH was dissolved in $10 \%$ methanol solution containing $0.5 \mathrm{mg} / \mathrm{mL}$ ascorbic acid. Afterwards $10 \%$ potassium ferrocyanide solution and $20 \%$ zinc acetate solution were added to the DWMH solution followed by centrifugation at 10,000 $\mathrm{g}$ for $5 \mathrm{~min}$. The supernatant was collected for HPLC assay. Processed samples of $10 \mu \mathrm{L}$ were injected into a HPLC system (Agilent 1260, USA) equipped with a Kromasil $\mathrm{C}_{18}$ column $(100-5-\mathrm{C} 18,4.6 \times 250 \mathrm{~mm}, 5 \mu \mathrm{m})$. The mobile phase comprised of $0.05 \%(\mathrm{v} / \mathrm{v})$ trifluoroacetic acid solution (A) and methanol (B), and flowed at 1 $\mathrm{mL} / \mathrm{min}$. The following linear gradient was used: hold at $22 \% \mathrm{~B}$ for $4 \mathrm{~min}$, increased to $60 \% \mathrm{~B}$ over 11 min, hold at $60 \% \mathrm{~B}$ over $6 \mathrm{~min}$, and then returned to the initial condition over $4 \mathrm{~min}$ and requilibrated for $5 \mathrm{~min}$. The absorption of elutes was monitored at $280 \mathrm{~nm}$ by a UV detector. 


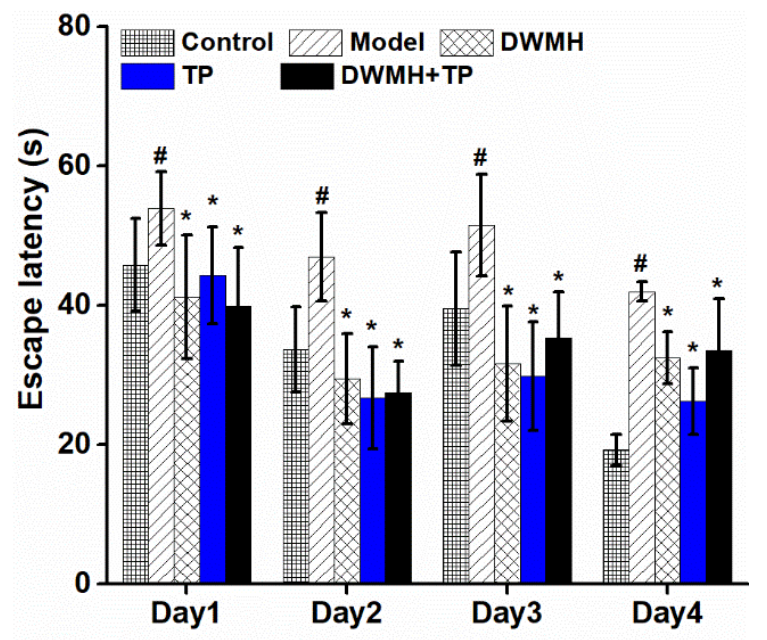

Figure S2. Escape latency over the training days of the Morris water maze experiment $(n=10)$. Effects of DWMH, TP alone or their combination on scopolamine-induced learning and memory deficits were evaluated in mice. Significant differences are marked as follows: *, $p<0.05$, compared to the model group; $\#, p<0.05$, compared to the control group. 

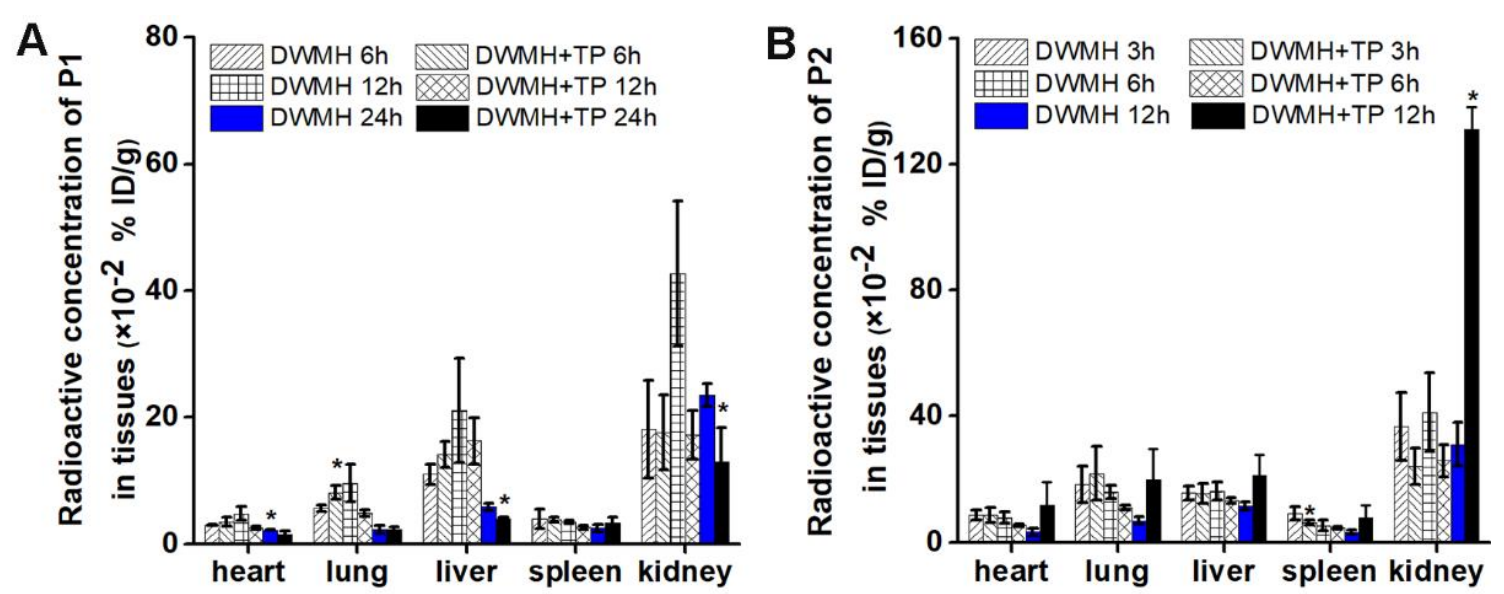

Figure S3. Distribution of ${ }^{99 \mathrm{~m}} \mathrm{Tc}-\mathrm{P} 1$ (A) or ${ }^{99 \mathrm{~m}} \mathrm{Tc}-\mathrm{P} 2$ (B) in mouse tissues after oral administration of ${ }^{99 \mathrm{~m}} \mathrm{Tc}-\mathrm{P} 1$ or ${ }^{99 \mathrm{~m}} \mathrm{Tc}-\mathrm{P} 2$ blended DWMH at a dose of $400 \mathrm{mg} / \mathrm{kg}(n=5)$. For the combinational treatment group, TP was orally coadministrated with DWMH to mice at a dose of $8 \mathrm{mg} / \mathrm{kg}$. Significant differences are marked as follows: ${ }^{*}, p<0.05$, compared to the DWMH group. 


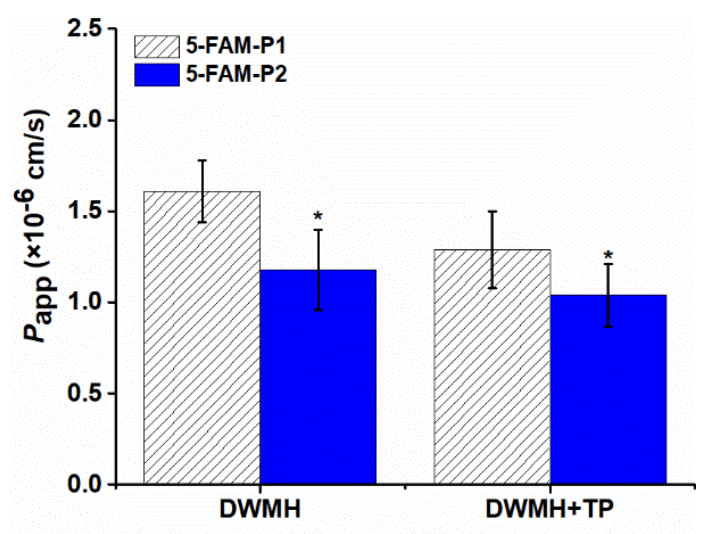

Figure S4. Apparent permeability coefficients $\left(P_{\text {app }}\right)$ of 5-FAM-P1 or 5-FAM-P2 through Caco-2 cell monolayers $(n=4-6)$. Significant differences are marked as follows: ${ }^{*}, p<0.05$, compared with the value of 5-FAM-P1. 
Table S1. HPLC assay of polyphenol ingredients in TP and DWMH.

\begin{tabular}{ccc}
\hline sample & constituent & content (\%) \\
\hline \multirow{2}{*}{$\mathrm{TP}$} & epicatechin & 5.158 \\
& epicatechin gallate & 15.531 \\
& epigallocatechin & 7.766 \\
& epigallocatechin gallate & 54.758 \\
\hline DWMH & epicatechin & $<0.0004$ \\
& epicatechin gallate & $<0.0002$ \\
& epigallocatechin & $<0.003$ \\
& epigallocatechin gallate & $<0.0005$ \\
\hline
\end{tabular}

